\title{
EFFECTIVENESS OF SODIUM DICHLOROACETATE AGAINST GLIOMA C6 DEPENDS ON ADMINISTRATION SCHEDULE AND DOSAGE
}

\author{
A.G. Fedorchuk, O.N. Pyaskovskaya, G.V. Gorbik, I.V. Prokhorova, D.L. Kolesnik, G.I. Solyanik* \\ R.E. Kavetsky Institute of Experimental Pathology, Oncologyand Radiobiology, NASof Ukraine, Kyiv 03022, Ukraine
}

Background: Anticancer action of sodium dichloroacetate (DCA) could be related to its ability to activate oxidative phosphorylation leading to enhanced generation of reactive oxygen species and induction of apoptosis. On the other hand, activation of oxidative phosphorylation could promote tumor cell survival, in particular, via increased ATP synthesis. Such ambiguous effects of DCA could influence its anticancer effectiveness, depending on biological properties of a tumor, schedule of DCA administration and its dosage. The aim of the study was to analyze anticancer effect of DCA against glioma C6 in rats under conditions of different schedules of its administration and various dosages. Materials and Methods: The study was carried out in Wistar rats with intracerebrally transplanted glioma C6 cells. Therapy with DCA was performed as follows: daily for 6 days starting from the second day after tumor cell transplantation (schedule I) or $7^{\text {th }}$ day (schedule II) at a dose of $1.0 \mathrm{~g} / \mathrm{kg}$, or daily for 13 days starting from the second day at doses of $1.0 ; 1.5 \mathrm{or} 4.5 \mathrm{~g} / \mathrm{kg}$ (schedule III). An influence of hypoxia on anticancer effect of DCA was studied using hypoxic chambers where oxygen content was maintained at a level of 12.5-13\% for $3 \mathrm{~h}$ after DCA administration to glioma C6 bearing rats. The state of mitochondrial electron transport chain components in tumor cells was studied using electron paramagnetic resonance. Results: It has been shown that therapy with DCA using schedule I resulted in 15\% decrease of animals life span (LS; $p<0.05$ ), while the use of schedule II had no effect on this index. Prolonged administration of DCA (schedule III) resulted in significant antitumor effect and increased LS of rats by $25.5 \%$ (p $<0.05)$. Under hypoxic conditions, treatment with DCA resulted in a significant increase of animal LS by 15-22\%. Dosage of DCA had a moderate effect of its anticancer action. Maximal effect, an increase of LS by 34.5\% ( $<<0.05)$ was detected at a dose of $1.5 \mathrm{~g} / \mathrm{kg}$. It has been shown that anticancer activity of DCA under all studied conditions is not related to its influence on a functional state of tumor cell mitochondria. Conclusion: Anticancer effect of DCA significantly depends on a schedule of its administration; being administered at equal total dose, but dependent on the schedule DCA could cause ambiguous effects varying from tumor growth stimulation to significant anticancer activity. Under hypoxic conditions, anticancer efficacy of DCA against glioma C6 is significantly enhanced.

Key Words: sodium dichloroacetate, glioma C6, mitochondrial electron transport chain.

According to statistics of World Health Organization, an average rate of brain tumors incidence is $10.9-12.8$ per 100000 of population [1]. Nearly $60 \%$ of all tumors of central nervous system are malignant neoplasmae, among which gliomas account up to $50-55 \%$. By statistical data, gliomas yield just $1.4 \%$ from all clinically diagnosed tumors, but the prognosis for glioma patients is the most unfavorable. An average survival time of the patients with malignant glioma after its primary diagnosis is approximately 8 months in Ukraine and 14 months in USA [2]. That's why the therapy of patients with gliomas of different grade of malignancy is among the most important problems of modern clinical oncology.

Despite the development of numerous novel anticancer means, at present time the main approach for therapy of glioma patients is still a "therapy of despair", i.e. surgery, butits effectiveness is insufficiently low $[3,4]$. It is supposed that inefficiency of radical removal of gliomas is largely caused by tumor invasion into normal surrounding tissues with the formation of indiscrete margins significantly reduces.

Modern protocols for radiotherapy of malignant glioma recommend a fractionated irradiation with low daily doses (>2 Gy), with a total dose of 60-90 Gy [5]. However, even

Submitted: May 17, 2016.

*Correspondence: E-mail: gsolyanik@gmail.com

Abbreviations used: DCA - sodium dichloroacetate; EPR - electron paramagnetic resonance; LS - life span; MtETC - mitochondrial electron transport chain; PDH - pyruvate dehydrogenase kinase; ROS - reactive oxygen species. the modern methods of irradiation could not help to overcome an extremely low effectiveness of the therapy, due to high radioresistance of glioma caused by the development of local hypoxia [6].

Chemotherapeutics (including target agents) are of low effectiveness in the case of gliomas due to their poor bioavailability [7-10]. For example, in the majority of cases analysis of biopsy from malignant glioma evidences on the presence of defective but functional hematoencephalic barrier which prevents the passage of many target preparations (especially antibodies against growth factors and receptors). For treatment of malignant glioma just one alkylating cytostatic preparation, temozolomide is used. One should note that a response to therapy with temozolomide could be observed just in 5-8\% patients in the case of monotherapy, and in approximately in $20 \%$ patients in the case of adjuvant therapy with temozolomide combined with radiotherapy or surgical treatment [11, 12].

It is known that in a large part of malignant tumors ATP is generated via glycolysis even in the presence of oxygen (Warburg's effect). Aerobic glycolysis is a low-effective way of ATP generation, but it supports high proliferative potential of tumor cells and promotes their survival decreasing the risk of formation of apoptotic stimuli [13, 14]. Therefore, in recent years tumor metabolism, which provides these growth benefits, is considered as a new target for anticancer therapy, and compounds capable to inhibit glycolysis in tumor cells are studied as potentially effective anticancer agents [15-17]. Among antimetabolic agents active against many cancer cell types one could mention sodium dichloroacetate (DCA) [18-20]. 
DCA inhibits pyruvate dehydrogenase kinase $(\mathrm{PDH})$ that results in indirect activation of enzymes of PDH complex and consequently in a shift of cell metabolism from glycolysis toward oxidative phosphorylation. In a case of activation of oxidative phosphorylation and decreased intensity of glycolysis upon DCA action, one could expect an inhibition of tumor cell proliferation caused, in particular, by significant decrease of the content of metabolites of pentose-phosphate bypass and pyruvate important for synthesis of proteins and nucleic acids. DCA-induced activation of mitochondrial metabolism may lead to blocking of tumor cell proliferation and enhancement of reactive oxygen species (ROS) generation and apoptosis induction. However, despite theoretic apparentness of anticancer action of DCA, its effectiveness is not guaranteed. As it has been shown [21], at a wide concentration range DCA exerts cytostatic but not cytotoxic action, what is supported by the data on an absence of its effect toward intracellular level of ROS, the rate of lactate production and apoptosis rate in glioma $\mathrm{C} 6$ cells.

In addition, it is necessary to note that DCA-induced activation of oxidative phosphorylation may result not only in anticancer effect but also may promote tumor cell survival via increased ATP synthesis. Such ambivalent influence on tumor cell survival predisposes a variability of DCA activity $[22,23]$. Biologic properties of a tumor, schedules of DCA administration and its doses could significantly affect its anticancer effectiveness.

The aim of the study was to analyze anticancer effectiveness of DCA against glioma C6 in rats under conditions of different schedules of its administration and dosing.

\section{MATERIALS AND METHODS}

The study was performed in female Wistar rats 2.5-3 months old weighting $90-150 \mathrm{~g}$, bred at animal facility of R.E. Kavetsky Institute of Experimental Pathology, Oncology and Radiobiology of the National Academy of Sciences of Ukraine (IEPOR). The use and care of the experimental animals have been performed in accordance with the standard international rules of biologic ethics and was approved by Institutional Animal Care and Use Committee. Glioma 66 cell line was obtained from the National Bank of Cell Lines and Tumor Strains of IEPOR.

Glioma C6 cells were cultured in vitro in DMEM culture medium supplemented with 10\% FBS (Sigma, USA), $2 \mathrm{mM}$ L-glutamine, and $40 \mathrm{mg} / \mathrm{ml}$ gentamycin at $37^{\circ} \mathrm{C}$ in humidified atmosphere with $5 \% \mathrm{CO}_{2}$. Transplantation of glioma $\mathrm{C} 6$ cells was performed under total anesthesia via intracerebral inoculation of $0.6 \cdot 10^{6}$ cells in $0.05 \mathrm{ml}$ of physiologic solution in a left parietal area (anterior horn of left lateral ventricle).

Aqueous DCA solution (Sigma-Aldrich, USA) was administered daily per os through a tube in a volume of $3 \mathrm{ml}$ per animal. Therapy with DCA was performed by different schedules and at different doses as follows:

- schedule I - DCA was administered daily for 6 days starting from the second day after tumor cell transplantation at a total dose of $1.0 \mathrm{~g} / \mathrm{kg}$;
- schedule II - DCA was administered daily for 6 days starting from the $7^{\text {th }}$ day after tumor cell transplantation at a total dose of $1.0 \mathrm{~g} / \mathrm{kg}$;

- schedule III - DCA was administered daily for 13 days starting from the second day at total doses of 1.0; 1.5 or $4.5 \mathrm{~g} / \mathrm{kg}$.

All animals from corresponding control groups received $3.0 \mathrm{ml}$ of water for injections by the schedules for DCA administration. There were 13-15 rats per each group.

At day 14 after tumor cell inoculation 4-5 rats from each group were sacrificed under ether narcosis, blood was collected; brain tissue was taken for further examination.

The changes in survival time (CST) of remaining animals served as an index of anticancer effect of the treatment calculated by a formula:

$$
\operatorname{CST}(\%)=100 \cdot\left(S T-S T_{C}\right) / S T_{C} \text {, }
$$

where $S T$ and $S T_{C}$ are survival time of rats from experimental and control groups, respectively.

An influence of hypoxia (inhibitor of oxidative phosphorylation) on anticancer effect of DCA was studied using hypoxic chambers where oxygen content was maintained at a level of $12.5-13 \%$ for $3 \mathrm{~h}$ after DCA administration to glioma $\mathrm{C} 6$ bearing rats. Content of oxygen in hypoxic chambers was controlled using oxymeter $\mathrm{ISO}_{2}$ (World Precision Instruments, USA). Anticancer effects of the treatment were evaluated by prolongation of survival time of animals with glioma $\mathrm{C} 6$.

The changes of the state of mitochondrial electron transport chain (MtETC) components in tumor cells were analyzed using the method of electron paramagnetic resonance. Electron paramagnetic resonance (EPR)-analysis of the samples was performed at $77 \mathrm{~K}$ using spectrophotometer E-109 Varian (USA). By the data of EPR spectroscopy, the levels of reduced non-heme iron-sulfur (Fe-S) centers ( $g=1.94)$ of MtETC proteins, nitrosyl (NO) complexes of heme iron $\left(g_{\text {cep }}=2.01\right)$ and Fe-S nitrosyl complexes $\left(g_{\text {cep }}=2.03\right)$ were determined.

Statistical analysis of the data was performed by descriptive methods, correlation analysis, non-linear regression analysis, Student's $t$-test, and Mann - Whitney Utest, with the use of Microsoft Excel, Microcal Origin and Statistica. The data are presented as $\mathrm{M} \pm \mathrm{m}$.

\section{RESULTS AND DISCUSSION}

The results of the study have shown that an effectiveness of DCA against glioma C6 depends on administration schedule and is significantly increased if the animals are kept under hypoxic conditions for $3 \mathrm{~h}$ after administration of this agent.

As one may see (Table 1), therapy with DCA by schedule I resulted in $15 \%$ decrease of animals life span (LS; $p<0.05$ ). Upon application of schedule II which differs from schedule I by later terms of treatment initiation, no effect on LS of experimental animals was observed. Prolonged administration of DCA (schedule III) resulted in significant antitumor effect and increased LS of rats by $25.5 \%(p<0.05)$.

Usually in preclinical trials an effectiveness of anticancer agents administration of which begins at the background of an already developed tumor (days 7-10 after 
tumor cell inoculation), is lower compared with that if such agents are administered at earlier terms (day 2 after tumor cell inoculation). However, our data have demonstrated an opposite picture: an efficacy of DCA is higher against a developed tumor. It is known that a developed tumor is characterized by the development of intratumoral hypoxia. An increase of LS of rats with glioma $\mathrm{C} 6$ after prolonged administration of DCA at the background of a developed tumor could be related with the development of intratumoral hypoxia as far as DCA-induced activation of oxidative phosphorylation under conditions of oxygen deficiency significantly decreases ATP synthesis and, consequently, may result in tumor cell death. This assumption is in agreement with the results of the study that analyzed an influence of hypoxia on an effectiveness of cytotoxic/ cytostatic action of DCA against glioma C6 cells in vitro. As it has been shown [21], significant enhancement of cytotoxic action of DCA against glioma 66 cells under hypoxic conditions (triple decrease of DCA IC $\mathrm{I}_{50}$ vs that under normoxic conditions of culturing) was determined by high-level DCA-induced necrosis of the cells.

Administration of DCA under hypoxic conditions resulted in significant increase of its anticancer effect. In particular, in the case of schedule I DCA administration under hypoxic conditions had no effect on LS of experimental animals while in an absence of hypoxia such therapy stimulated tumor process and caused 15\% reduction of LS. Being administered by schedule II under hypoxic conditions, DCA significantly prolonged LS of rats by $22 \%$ compared to the animals treated with DCA under conditions of normoxia (Table 1). However, it is necessary to note that significant variability of antiglioma action of DCA upon different schedules of its administration was observed under conditions of hypoxia as well as normoxia.

Table 1. Anticancer effectiveness of DCA administered by different schedules under conditions of normoxia and hypoxia

\begin{tabular}{lcccc}
\hline \multirow{2}{*}{ Type of therapy } & \multicolumn{2}{c}{ Schedule of DCA administration } & Change \\
\cline { 2 - 4 } & Number & $\begin{array}{c}\text { Beginning } \\
\text { (day) }\end{array}$ & $\begin{array}{c}\text { Duration of treat- } \\
\text { ment (days) }\end{array}$ & $\begin{array}{c}\text { of LS, \% } \\
\text { DCA }\end{array}$ \\
\hline II & $2^{\text {nd }}$ & 6 & -15.0 \\
& II & $7^{\text {th }}$ & 6 & 0.0 \\
DCA + hypoxia & III & $2^{\text {nd }}$ & 13 & +25.5 \\
& II & $2^{\text {nd }}$ & 6 & 0.0 \\
& $7^{\text {th }}$ & 6 & +22.0 \\
\hline
\end{tabular}

Among possible mechanisms of anticancer action of inhibitors of energetic metabolism of tumor cells, including DCA, one could mention an ability of these agents to exert a damaging effect on a functional state of MtETC in tumor cells through activation of oxidative phosphorylation, hypoxia and possible defectiveness of mitochondrial system. That's why we have studied an influence of DCA on a functional state of MtETC using EPR spectroscopy.

High invasiveness of glioma complicates an accurate separation of tumor and normal tissues. That's why for preparation of EPR samples we have used the tissue of cerebral hemisphere with the tumor, and for a comparison - the tissue of cerebral hemisphere without tumor.

As indexes of functional state of MtETC we have used the levels of NO complexes of mitochondrial heme iron (that reflects a damaging action of ROS and nitrogen toward ETC), and the content of Fe-S centers, directly reflecting a functional state of this energetic system.
An analysis of DCA effects under conditions of normoxia and hypoxia on a functional state of MtETC in glioma C6 cells revealed an absence of correlation between MtETC functionality and LS of experimental animals (Table 2).

As shown in Table 2, administration of DCA by all schedules under conditions of normoxia and hypoxia had no effect on the levels of Fe-S centers in cerebral hemisphere with glioma $\mathrm{C} 6$ even at the background of significant (more than $46.7 \%$ ) increase of nitrosylation level of heme proteins registered after DCA administration under normoxic conditions. It is supposed that a decrease of the content of Fe-S centers in tumor tissue characterizes a malfunction of MtETC resulting in tumor cell death. Unchanged content of Fe-S centers indicated that anticancer action of DCA is not related to its influence on a functional state of mitochondria in glioma C6 cells.

Table 2. Influence of DCA administered by different schedules under conditions of normoxia and hypoxia on the indexes of a functional state of MtETC in the cells of cerebral hemisphere with glioma

\begin{tabular}{|c|c|c|c|}
\hline \multirow{2}{*}{ Group of animals } & \multicolumn{3}{|c|}{ Cerebral hemisphere with glioma } \\
\hline & Schedule I & Schedule II & Schedule III \\
\hline \multicolumn{4}{|c|}{ Intensity of EPR signal for NO-heme complexes ( $g=2.01$; a.u.) } \\
\hline & $100.0 \pm 2.9$ & 100. & 100.0 \\
\hline & $21.7^{\star}$ & 146 & $0.8^{*}$ \\
\hline DCA + hypoxia & $87.4 \pm 14.3$ & $148.4 \pm 9.8^{*}$ & 0.7 \\
\hline \multicolumn{4}{|c|}{ Intensity of EPR signal for Fe-S centers ( $g=1.94$; a.u.) } \\
\hline Control & $100.0 \pm 40.4$ & $100.0 \pm 19.1$ & 100 \\
\hline & $97.5 \pm 17.4$ & 112 & $83.3 \pm 19.2$ \\
\hline DCA + hypoxia & $100.6 \pm 37.3$ & $115.1 \pm 40.0$ & $108.3 \pm 16.0$ \\
\hline
\end{tabular}

Note: ${ }^{\star} \mathrm{p}<0.05$, differences are significant as compared to the value for control.

Contrary to the brain tissue samples with glioma, the content of NO-heme complexes in normal brain tissue after DCAadministration by schedules I and III significantly decreased by more than $17 \%$ under conditions of normoxia and hypoxia (Table 3). However, the level of Fe-S centers in normal brain tissue remained unaltered.

Table 3. Influence of DCA administered by different schedules under conditions of normoxia and hypoxia on the indexes of functional state of MtETC in the cells cerebral hemisphere without glioma

\begin{tabular}{|c|c|c|c|}
\hline \multirow{2}{*}{ Group of animals } & \multicolumn{3}{|c|}{ Cerebral hemisphere without glioma } \\
\hline & Schedule I & Schedule II & Schedule III \\
\hline \multicolumn{4}{|c|}{ Intensity of EPR signal for NO-heme complexes ( $\mathrm{g}=2.01$; a.u.) } \\
\hline Control & $100.0 \pm 3.4$ & $100.0 \pm 4.3$ & 100.0 \\
\hline DCA & $82.8 \pm 3.4^{*}$ & $119.6 \pm 2.9^{*}$ & $82.9 \pm 3.6^{\star}$ \\
\hline DCA + hypoxia & $72.1 \pm 9.9^{\star}$ & $123.0 \pm 9.1$ & $56.8 \pm 20.4^{\star}$ \\
\hline \multicolumn{4}{|c|}{ Intensity of EPR signal for Fe-S centers ( $g=1.94 ;$ a.u.) } \\
\hline Control & $100.0 \pm 34.0$ & $100.0 \pm 5.3$ & $100.0 \pm 20.0$ \\
\hline DCA & $129.0 \pm 37.7$ & $103.2 \pm 23.8$ & $101.3 \pm 28.1$ \\
\hline DCA + hypoxia & $109.9 \pm 17.3$ & $105.3 \pm 21.2$ & $104.0 \pm 19.0$ \\
\hline
\end{tabular}

Note: ${ }^{*} p<0.05$, differences are significant as compared to the value for control.

The study of effects of DCA dosing on LS of rats was done by the schedule with the highest anticancer efficacy. It has been shown that DCA dose moderately affected an efficacy of its antiglioma action (Figure, Table 4). The maximal effect was registered if DCA was administered at a dose of $1.5 \mathrm{~g} / \mathrm{kg}$ : LS of experimental animals increased by $34.5 \%(p<0.05)$. An elevation of DCA dose up to $4.5 \mathrm{~g} / \mathrm{kg}$ did not lead to the further rise of its efficacy: the LS of rats was significantly higher than that in control animals not treated with DCA, but didn't differ from that in animals treated with a dose of $1.0 \mathrm{~g} / \mathrm{kg}$.

An analysis of DCA effects upon its prolonged administration at different doses did not reveal significant changes in the nitrosylation levels of heme proteins and 
content of Fe-S centers in the cells of cerebral hemisphere with glioma (Table 5), and normal brain tissue (Table 6).

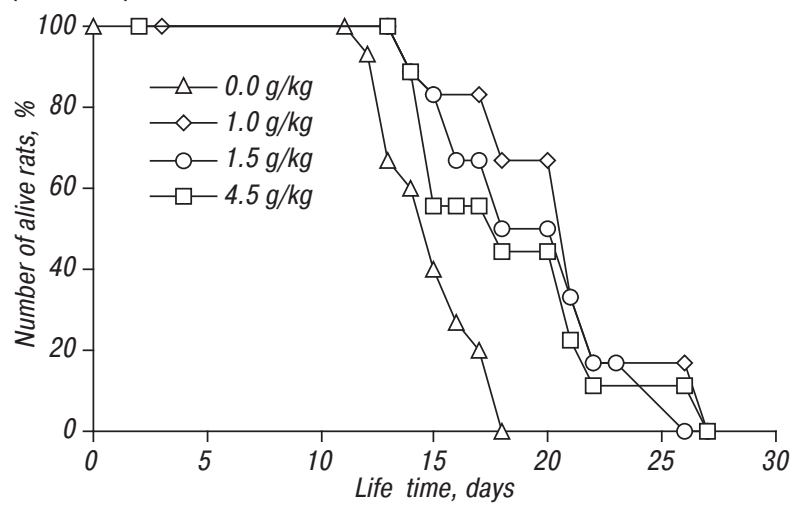

Figure. Survival curves of rats with glioma C6 treated with DCA administered at different doses by schedule III

Table 4. Dependence between DCA dosing and its antiglioma effectiveness Total dose, Schedule of ad- Beginning Duration of ther- Change of LS,

\begin{tabular}{ccccc}
$\mathrm{g} / \mathrm{kg}$ & ministration & (day) & apy (days) & $\%$ \\
\hline 1.0 & III & $2^{\text {nd }}$ & 13 & +25.5 \\
1.5 & III & $2^{\text {nd }}$ & 13 & +34.5 \\
\hline
\end{tabular}

Table 5. Effects of DCA administered at different doses on the indexes of a functional state of MtETC in the cells of cerebral hemisphere with glioma

\begin{tabular}{|c|c|c|c|}
\hline \multirow{2}{*}{ Group of animals } & \multicolumn{3}{|c|}{ Cerebral hemisphere with glioma } \\
\hline & DI & D2 & $\overline{\mathrm{D} 3}$ \\
\hline \multicolumn{4}{|c|}{ Intensity of EPR signal for NO-heme complexes ( $g=2.01$; a.u.) } \\
\hline Control & $100.0 \pm 1.4$ & $100.0 \pm 3.5$ & $100.0 \pm 3.5$ \\
\hline DCA & $152.4 \pm 20.8^{*}$ & $93.1 \pm 13.0$ & $103.0 \pm 3.1$ \\
\hline \multicolumn{4}{|c|}{ Intensity of EPR signal for Fe-S centers ( $g=1.94$; a.u.) } \\
\hline Control & $100.0 \pm 28.9$ & $100.0 \pm 5.5$ & $100.0 \pm 5.5$ \\
\hline DCA & $83.3 \pm 19.2$ & $88.1 \pm 12.1$ & $101.2 \pm 10.6$ \\
\hline
\end{tabular}

Note: ${ }^{\star} p<0.05$, differences are significant as compared to the value for control.

Table 6. Effects of DCA administered at different doses on the indexes of a functional state of MtETC in the cells of cerebral hemisphere without glioma

\begin{tabular}{|c|c|c|c|}
\hline \multirow{2}{*}{ Group of animals } & \multicolumn{3}{|c|}{ Cerebral hemisphere without glioma } \\
\hline & $\mathrm{DI}$ & $\mathrm{D} 2$ & D3 \\
\hline \multicolumn{4}{|c|}{ Intensity of EPR signal for N0-heme complexes ( $g=2.01$; a.u.) } \\
\hline Control & $100.0 \pm 2.7$ & $100.0 \pm 13.0$ & $100.0=$ \\
\hline DCA & $82.9 \pm 3.6^{\star}$ & $94.2 \pm 2.7$ & $79.4 \pm$ \\
\hline \multicolumn{4}{|c|}{ Intensity of EPR signal for Fe-S centers ( $g=1.94$; a.u.) } \\
\hline Control & $100.0 \pm 20.0$ & $100.0 \pm 15.5$ & $100.0 \pm 1$ \\
\hline DCA & $101.3 \pm 28.1$ & $118.6 \pm 3.6$ & $102.4 \pm 6.9$ \\
\hline
\end{tabular}

Note: ${ }^{*} p<0.05$, differences are significant as compared to the value for control.

An absence of such changes along with a high anticancer efficacy of DCA (especially at a dose of $1.5 \mathrm{~g} / \mathrm{kg}$ ) once more demonstrated that effects of DCA against glioma $\mathrm{C} 6$ have no relation to a functional state of tumor cell mitochondria.

In conclusion, the results of our study have shown that anticancer effectiveness of DCA significantly depends on the schedule of its administration whereupon it varies from a significant decrease of LS directly correlating with stimulation of tumor growth, to significant anticancer effect and increased LS of rats by $25.5 \%$. Prolonged metronomic administration of DCA provides better anticancer effectiveness with lowvariability between the doses. Also, it has been demonstrated that hypoxic conditions of animal keeping significantly elevate an antiglioma efficacy of DCA. Along with this, anticancer activity of this agent is not related to its effects on a functional state of tumor cell mitochondria.

\section{REFERENCES}

1. Weller M, Wick W, Aldape K, et al. Glioma. Nat Rev Dis Primers 2015; 1: 15017. doi: 10.1038/nrdp.2015.17.

2. Zozulya YuA, Vasil'ev IG, Glavacky AYa, et al. Brain gliomas. Current state of the problem and trends of further researches. Zozulya YuA, ed. Kyiv: ExOb, 2007. 631 p. (in Russian).

3. Aghi MK, Nahed BV, Sloan AE, et al. The role of surgery in the management of patients with diffuse low grade glioma: Asystematic review and evidence-based clinical practice guideline. J Neurooncol 2015; 125: 503-30.

4. Hervey-Jumper SL, Berger MS. Technical nuances of awake brain tumor surgery and the role of maximum safe resection. J Neurosurg Sci 2015; 59: 351-60.

5. Laperriere N, Zuraw L, Cairncross G. Radiotherapy for newly diagnosed malignant glioma in adults: a systematic review. Radiother Oncol 2002; 64: 259-73.

6. Ryken TC, Parney I, Buatti J, et al. The role of radiotherapy in the management of patients with diffuse low grade glioma: A systematic review and evidence-based clinical practice guideline. J Neurooncol 2015; 125: 551-83.

7. Norden AD, Drappatz J, Wen PY. Novel anti-angiogenic therapies for malignant gliomas. Lancet Neurol 2008; 7: 1152-60.

8. Huang TT, Sarkaria SM, Cloughesy TF, Mischel PS. Targeted therapy for malignant glioma patients: lessons learned and the road ahead. J Neurother 2009; 6: 500-12.

9. Staedtke V, Bai RY, Laterra J. Investigational new drugs for brain cancer. Expert Opin Investig Drugs 2016; 17: 1-20.

10. Kang JH, Adamson C. Novel chemotherapeutics and other therapies for treating high-grade glioma. Expert Opin Investig Drugs 2015; 24: 1361-79.

11. Baritchii A, Jurj A, Soritau O, et al. Sensitizer drugs for the treatment of temozolomide-resistant glioblastoma. J BUON 2016; 21: 199-207.

12. Mallick S, Gandhi AK, Rath GK. Therapeutic approach beyond conventional temozolomide for newly diagnosed glioblastoma: Review of the present evidence and future direction. Indian J Med Paediatr Oncol 2015; 36: 229-37.

13. Gatenby RA, Gillies RJ. Why do cancers have high aerobic glycolysis? Nat Rev Cancer 2004; 4: 891-9.

14. Kim JW, Dang CV. Multifaceted roles of glycolytic enzymes. Trends Biochem Sci 2005; 30: 142-50.

15. Jin S, DiPaola RS, Mathew R, White E. Metabolic catastrophe as a means to cancer cell death. J Cell Sci 2007; 3: 379-83.

16. Seyfried TN, Kiebish MA, Marsh J. Metabolic management of brain cancer. Biochim Biophys Acta 2011; 6: 577-94.

17. Decollogne S, Dilda PJ, Hau E, et al. Dual-targeting of aberrant glucose metabolism in glioblastoma. J Exp Clin Cancer Res 2015; 34: 14.

18. Kankotia S, Stacpoole PW. Dichloroacetate and cancer: new home for an orphan drug? Biochim Biophys Acta 2014; 1846: $617-29$.

19. Stacpoole PW. The dichloroacetate dilemma: environmental hazard versus therapeutic goldmine - both or neither? Environ Health Perspect 2011; 119: 155-8.

20. Michelakis ED, Webster L, Mackey JR. Dichloroacetate (DCA) as a potential metabolic-targeting therapy for cancer. Br J Cancer 2008; 99: 989-94.

21. Kolesnik DL, Pyaskovskaya ON, Boichuk IV, Solyanik GI. Hypoxia enhances antitumor activity of dichloroacetate. Exp Oncol 2014; 36: 231-5.

22. James MO, Stacpoole PW. Pharmacogenetic considerations with dichloroacetate dosing. Pharmacogenomics 2016; doi:10.2217/pgs-2015-0012.

23. Dunbar EM, Coats BS, Shroads AL, et al. Phase 1 trial of dichloroacetate (DCA) in adults with recurrent malignant brain tumors. Invest New Drugs 2014; 32: 452-64. 